\title{
An experimental model for the study of well-differentiated and dedifferentiated liposarcoma; deregulation of targetable tyrosine kinase receptors
}

\author{
Tingsheng Peng ${ }^{1,2}$, Pingyu Zhang ${ }^{1,2}$, Jeffery Liu ${ }^{1,2}$, Theresa Nguyen ${ }^{1,2}$, Svetlana Bolshakov ${ }^{1,2}$, Roman Belousov ${ }^{1,2}$, \\ Eric D Young ${ }^{1,2}$, Xiaoke Wang ${ }^{3}$, Kari Brewer ${ }^{1,2}$, Delores H López-Terrada ${ }^{4}$, Andre M Oliveira ${ }^{3}$, Alexander J Lazar ${ }^{2,5,6}$ \\ and Dina Lev $2,6,7$
}

Therapeutic progress in well-differentiated/dedifferentiated liposarcoma (WDLPS/DDLPS) is hampered by lack of relevant experimental models, thereby limiting comprehensive molecularly based investigations. Our goal is to bridge this experimental gap by establishing and characterizing an in vitro/in vivo model useful for examining WDLPS/DDLPS molecular pathogenesis and also therapeutic screening and testing. WDLPS/DDLPS cells were isolated from freshly resected human surgical specimens and were phenotypically and molecularly characterized. MDM2 amplification was determined via FISH analysis. Adipogenic differentiation was evaluated using Oil Red O staining and western blotting (WB). Tyrosine kinase receptors' (TKRs) expression in pre-adipocytes, adipocytes, WDLPS, and DDLPS cells was determined via western blot analysis. SCID mouse xenograft growth was assessed after subcutaneous and/or intraperitoneal tumor cell injection. There was enhanced proliferation, migration, invasion, survival, and pro-angiogenic capacity in DDLPS cells vs WDLPS cells. DDLPS cells formed tumors in SCID mice whereas WDLPS did not. WDLPS/DDLPS cells, especially those that exhibited baseline PPAR expression, partially retained terminal adipogenic differentiation capacity. MDM2 amplification was found in all WDLPS/DDLPS cell strains, CDK4 overexpression was observed in LPS cells as compared with normal adipocytes, and enhanced JUN expression and phosphorylation was seen in DDLPS cells as compared with WDLPS cells. The TKRs: MET, AXL, KIT, and IGF-1R were overexpressed in LPS cells vs normal adipocytes and pre-adipocytes. In conclusion, these newly established cellular and xenograft models can facilitate investigation of liposarcomagenesis, dedifferentiation, and tumor progression. Further studies of the molecular deregulations so identified may lead to improved therapeutic strategies for patients afflicted by these unfavorable malignancies.

Laboratory Investigation (2011) 91, 392-403; doi:10.1038/labinvest.2010.185; published online 8 November 2010

KEYWORDS: adipogenesis; dedifferentiated liposarcoma; preclinical experimental model; targeted therapy; tyrosine kinase receptors; well-differentiated liposarcoma

The adipogenic-origin well-differentiated and dedifferentiated liposarcoma (WDLPS and DDLPS) together constitute the most common soft tissue sarcoma histological subtypes. ${ }^{1}$ WDLPS is a non-metastasizing tumor that often recurs after surgical resection. ${ }^{1,2}$ These tumors can develop within any deep-seated bodily location, yet demonstrate a predilection for the retroperitoneum, where repeated recurrences are highly morbid and even fatal. DDLPS, originally described by Evans in $1979,{ }^{3}$ is a biphasic tumor consisting of a WDLPS component juxtaposed to either a high-grade undifferentiated sarcoma with malignant fibrous histiocytoma or fibrosarcoma-like features or with a lower-grade sarcoma having the appearance of myxofibrosarcoma. ${ }^{1,4,5}$ About $90 \%$ of DDLPS are diagnosed as a component of a primary presenting lesion, whereas $10 \%$ are identified in the context of a recurrent tumor. ${ }^{6,7}$ DDLPS are

\footnotetext{
${ }^{1}$ Department of Surgical Oncology, The University of Texas MD Anderson Cancer Center, Houston, TX, USA; ${ }^{2}$ The Sarcoma Research Center, The University of Texas MD Anderson Cancer Center, Houston, TX, USA; ${ }^{3}$ Department of Laboratory Medicine and Pathology, Mayo Clinic, Rochester, MN, USA; ${ }^{4}$ Department of Pathology, Texas Children's Hospital and Baylor College of Medicine, Houston, TX, USA; ${ }^{5}$ Department of Pathology, The University of Texas MD Anderson Cancer Center, Houston, TX, USA; ${ }^{6}$ The Graduate School of Biomedical Sciences, Houston, TX, USA and ${ }^{7}$ Department of Cancer Biology, The University of Texas MD Anderson Cancer Center, Houston, TX, USA

Correspondence: Dr D Lev, MD, Department of Cancer Biology, MD Anderson Cancer Center, 8515 Fannin, Unit 1104, Houston, TX 77030, USA.

E-mail: dlev@mdanderson.org

Received 9 August 2010; revised 22 September 2010; accepted 22 September 2010
} 
significantly more aggressive than pure WDLPS, exhibiting a local recurrence rate of $>80 \%$, a distant metastasis rate of up to $20 \%$, and a five-year disease-specific survival rate of $40-60 \%$ despite an aggressive surgical approach combined with systemic chemotherapy. ${ }^{8}$ It is currently unresolved whether WDLPS and DDLPS comprise a disease continuum where dedifferentiation is a time-dependent phenomenon or alternatively whether these are two distinct and separate malignancies arising in different adipogenic-lineage cells of origin that share certain common molecular aberrations. ${ }^{9,10}$ Intriguing as a molecular question, resolving this debate also has significant clinical implications that will better inform therapeutic decision making in this disease. Whatever the resolution of this controversy, it is unequivocally certain that current therapeutic approaches for either WDLPS or DDLPS are insufficient given the marked rates of disease- and treatment-related morbidity and mortality. A better understanding of the molecular forces driving liposarcomagenesis, tumor progression, and dedifferentiation is necessary in order to develop more effective anti-LPS therapeutic strategies.

Over the past several decades, several molecular aberrations unique to WDLPS/DDLPS have been identified. It is now known that these tumors contain supernumerary ring chromosomes and/or giant marker chromosomes composed (exclusively or partially) of amplified genomic sequences derived from chromosome $12 \mathrm{q} 13-\mathrm{q} 15 .{ }^{11}$ Overexpression of genes included in this interval (eg MDM2 and CDK4 and their cognate protein products) has been extensively validated, enhancing current LPS diagnostic paradigms; ${ }^{12}$ furthermore, a role for these proteins in tumorigenesis has been suggested. ${ }^{13,14}$ With the advent of high-throughput, high-resolution techniques such as array CGH and cDNA expression profiling, a growing number of potential LPSassociated molecular deregulations have been recently identified in frozen or paraffin-embedded tumor specimens. ${ }^{15-17}$ Translating these tissue-based observations into mechanistically driven molecular biology insights leading to preclinical studies that can impact patient management is the crucially needed next step. Towards that end, reproducible WDLPS/DDLPS experimental models recapitulating the clinical behavior of these unique malignancies in vitro and in vivo are essential, and their paucity has been a major limitation to incisive and comprehensive LPS-dedicated research. Illustrative of this fundamental lack of relevant LPS research resources, the most widely utilized commercially available human LPS cell line is SW872 (ATCC), which lacks MDM2 amplification, a hallmark of LPS tumors. ${ }^{18}$

The goal of the current study was to bridge the above experimental gap by (1) establishing a model of LPS useful for WDLPS and DDLPS molecular pathogenesis studies and for in vitro/in vivo screening and testing of novel, potentially efficacious therapeutics; (2) identifying functional differences between WDLPS and DDLPS; and (3) determining common, therapeutically targetable, WDLPS/DDLPS tyrosine kinase receptor (TKR) deregulations.

\section{MATERIALS AND METHODS \\ Cell Lines/Strains and Reagents}

The previously established human DDLPS cell line LPS141 was kindly provided by Dr Jonathan Fletcher (Brigham and Women's Hospital, Boston, MA, USA ${ }^{19}$ ) human dermal microvascular endothelial cells and human white pre-adipocytes (HWP) primary cultures were purchased from PromoCell (Heidelberg, Germany). Human umbilical vein endothelial cells (HUVECs) were purchased from ATCC. HWP were differentiated into adipocytes per company's instructions using a commercial pre-adipocyte differentiation media (DM) (serum-free media containing: insulin, dexamethasone, IBMX, L-thyroxine, ciglitazone, and heparin) and adipocyte nutrition media (3\% FCS supplemented media containing: insulin, dexamethasone, and IBMX). Adipogenic differentiation was confirmed via Oil red $\mathrm{O}$ staining as previously described. ${ }^{20}$

\section{Liposarcoma cells isolation}

This procedure was conducted with the approval from the Institutional Review Board at The University of Texas M.D. Anderson Cancer Center and patient's informed consent. Tumor cell isolation was conducted as previously described. $^{21}$ Briefly, fresh sterile samples from surgically resected tumors were minced in culture medium and then digested via incubation with collagenase type I (3\%), DNase I $(0.02 \%)$, and hyaluronidase $(1.5 \mathrm{mg} / \mathrm{ml})$ at $37^{\circ} \mathrm{C}$ for $2-4 \mathrm{~h}$. The sample was strained through a wire mesh screen, and undigested tissue was discarded. After centrifugation, washes, and resuspension in PBS, the sample was gently transferred to Histopaque tubes containing $10 \mathrm{ml}$ Histopaque (100\%; Sigma) overlayed with $15 \mathrm{ml}$ of Histopaque (75\%). The tubes were then centrifuged at $40^{\circ} \mathrm{C}$ for $30 \mathrm{~min}$ at $1200 \mathrm{~g}$. After centrifugation, tumor cells located in the top interface (over the $75 \%$ Ficoll) were collected and plated (high fat containing cells have been discarded). Cells were cultured and passaged in DMEM supplemented with glucose and 10\% FBS.

Commercially available antibodies were used for immunoprecipitation, western blotting (WB) analysis, or immunohistochemical detection of: CEBP $\alpha$, PPAR $\gamma$, phospho-JUN, JUN, CDK4, MET, AXL, HER-2, RET, PDGFRA, PDGFRB (Cell Signaling, Beverly, MA, USA); Ki-67 (Dako, Carpenteria, CA, USA); CD31 (PharMingen, San Diego, CA, USA); EGFR, ROR2, IGF-Ir $\alpha$, and $\beta$-actin (Santa Cruz Biotechnology, Santa Cruz, CA, USA); and KIT (Stressgen, Ann Arbor, MI, USA).

\section{MDM2 FISH Analysis}

FISH was performed on fixed cultured cells and FFPE tissues with a laboratory-developed BAC label probe cocktail using BAC clones; RP11-185H13, RP11-450G15, RP11-816C9, RP11-630N19, RP11-717F7, RP11-1104N20 and RP11- 
426B12, purchased from the Children's Hospital Oakland research Institute, Oakland, CA, USA, specific for the 12q15 region (Spectrum orange) and a probe specific for the centromeric region of chromosome 12 (spectrum green; Abbott Molecular, DesPlaines, IL, USA), as previously described. ${ }^{22}$ A minimum of 100 nuclei per slide were analyzed. The average number of MDM2 and CEP12 signals was then determined and a $M D M 2 / \mathrm{CEP} 12$ ratio was calculated for each case. A ratio $\geq 2$ was considered amplified for the MDM2 gene, whereas a ratio $<2.0$ was considered nonamplified. A ratio of $<2.0$ with $>2$ signals of both probes was considered polysomic for CEP12. The established DDLPS cell line LPS-141 and normal adipocytes were used as positive and negative controls, respectively.

\section{Short Tandem Repeat DNA Fingerprinting}

DNA fingerprinting was done on cultured cells and their tumor of origin as previously described. ${ }^{21}$

\section{Western Blot Analysis and Immunoprecipitation}

Western blot analysis was performed by standard methods. Briefly, $25-50 \mu \mathrm{g}$ of proteins extracted from cultured cells were separated by SDS-PAGE and transferred onto nitrocellulose membranes. Membranes were blocked with milk or BSA and blotted with relevant antibodies. HRPconjugated secondary antibodies were detected by enhanced chemiluminescence (Amersham Biosciences, Pittsburgh, PA, USA). For immunoprecipitation, protein lysates $(500-1000 \mu \mathrm{g})$ prepared from cultured cells were used. Immunocomplex pull down was achieved via overnight incubation of protein lysates with relevant antibodies bound to protein G Sepharose beads (GE Healthcare) at $4{ }^{\circ} \mathrm{C}$. After careful washing, loading buffer (Bio-Rad) was added, and the samples were boiled at $100{ }^{\circ} \mathrm{C}$ for $6 \mathrm{~min}$. Coimmunoprecipitated proteins were then subjected to $\mathrm{WB}$ as described above.

\section{Growth Assays}

\section{MTS assays}

MTS assays were conducted using CellTiter96 Aqueous Non-Radioactive Cell Proliferation Assay kit (Promega, Madison, WI, USA), as per the manufacturer's instructions. Absorbance was measured at a wavelength of $490 \mathrm{~nm}$, and the absorbance values of treated cells are presented as a percentage of the absorbance of untreated cells.

\section{Colony formation assay}

One hundred viable cells per well were plated and allowed to grow in normal medium for 10 days and then stained for $30 \mathrm{~min}$ at room temperature with a $6 \%$ glutaraldehyde, $0.5 \%$ crystal violet solution. Pictures were captured digitally and colonies were counted. Anchorage-independent growth: $1 \times 10^{3}$ viable cells were plated in a 24 -well plate in culture medium containing $0.35 \%$ agarose overlying a $0.7 \%$ agarose layer. Cells were incubated for 3 weeks at $37^{\circ} \mathrm{C}$. Cells were stained with $p$-iodonitrotetrazolium violet $(1 \mathrm{mg} / \mathrm{ml})$ for $24 \mathrm{~h}$ at $37^{\circ} \mathrm{C}$. Number of colonies per well were counted. All experiments were repeated three times for each cell strain/line.

\section{Migration and Invasion Assays}

Migration and invasion assays were conducted as described previously. ${ }^{21}$ BioCoat cell culture inserts and polycarbonate filters with $8 \mu \mathrm{m}$ pores (Becton Dickinson Labware, Franklin Lakes, NJ, USA) in 24-well tissue culture plates were used for modified Boyden chamber migration assays. Lower chamber compartments contained DMEM supplemented by $1 \%$ bovine serum albumin or $1 \%$ fetal bovine serum as chemoattractants. Cells $\left(5 \times 10^{4}\right)$ were seeded in the upper compartment and incubated at $37^{\circ} \mathrm{C}$ in a humidified atmosphere of $95 \%$ air and $5 \% \mathrm{CO}_{2}$. Invasion assays were conducted similarly using 24-well BioCoat Matrigel invasion chambers with $8 \mu \mathrm{m}$ pore size polycarbonate filters coated with Matrigel (Becton Dickinson Labware). After incubation, filters were fixed with $4 \%$ formaldehyde and stained with $0.2 \%$ crystal violet (Baxter Healthcare, Houston, TX, USA). Cells on the upper surface of the filters were removed by wiping with a cotton swab, and migratory and invasive activities were determined by counting the number of cells per high-power field $(\times 200)$ that had migrated to the lower side of the filter.

\section{In Vivo Gelfoam Angiogenesis Assay}

These experiments were approved by the MD Anderson Cancer Center Institutional Animal Care and Usage Committee. Gelfoam sponges (Pharmacia \& Upjohn, Peapack, NJ, USA) were cut into $\sim 0.50 .5 \mathrm{~cm}^{2}$ fragments and saturated overnight in $\mathrm{PBS}$ at $4{ }^{\circ} \mathrm{C}$. The next day, the sponges were placed on sterile filter paper to allow excess PBS to be drawn out. Sponges were incubated with conditioned media from LPS (WDLPS or DDLPS) cells. The sponges were allowed to sit at room temperature for $\sim 1 \mathrm{~h}$ and then implanted subcutaneously into the flank of SCID mice, as previously described. ${ }^{21}$ After 14 days, the gelfoam sponges were harvested and frozen in OCT (Sakura Fineter, Torrance, CA, USA). The frozen samples were later sectioned and probed for CD31.

\section{In Vivo Growth}

All animal procedures and care were approved by the MD Anderson Cancer Center Institutional Animal Care and Usage Committee. Animals received humane care as per the Animal Welfare Act and the NIH 'Guide for the Care and Use of Laboratory Animals.' WDLPS and DDLPS cells $\left(2 \times 10^{6} \%\right.$ $0.1 \mathrm{HBSS} /$ mouse) were injected subcutaneously into the flank of 6-week-old female hairless SCID mice. Mice were followed for tumor growth. Study was terminated when tumors reached $1.5 \mathrm{~cm}$ in largest dimension. Tumors were then resected, preserved in buffered formalin, and paraffin embedded. $\mathrm{H}+\mathrm{E}$ staining was done to evaluate tumor morphology. Immunohistochemical analysis for Ki-67 and CD31 was conducted as previously described. ${ }^{21}$ 


\section{Statistical Analysis}

Cell culture based assays were repeated at least three times and mean \pm s.d. was calculated. Cell lines were examined separately. For outcomes that were measured at a single time point, two-sample $t$ tests were used to assess the differences. Significance was set at $P \leq 0.05$.

\section{RESULTS}

WDLPS/DDLPS Tumor Cells Isolated from Fresh Surgical Specimens Exhibit Growth in Culture

Twenty-four WDLPS and 13 DDLPS surgical specimens were processed between January and December 2009. Only MDM2 + confirmed samples were used; all tumors originated in the retroperitoneum, abdomen, or pelvis. Using the Ficoll method for tumor cell isolation, high fat containing normal cells were excluded. The plating efficiency of WDLPS was $50 \%$, resulting in a total of 12 different $M D M 2+$ WDLPS primary cultures/cell strains (Table 1; Figure 1). Two of these cell strains (Lipo355 and Lipo723) were obtained from patients with a previous history of DDLPS. Plating efficiency of DDLPS cells was similar, with $8 / 13$ samples confirmed to consist of MDM2+ cells (Table 1; Figure 1).
Two of the cell strains obtained from DDLPS samples (Lipo203 and Lipo815) represented the well-differentiated component of these tumors. The morphology, Oil red O staining pattern, and MDM2 FISH analysis of WDLPS and DDLPS cells, as compared with pre-adipocytes and adipocytes, are depicted in Figures $1 \mathrm{~b}$ and c. A small fraction $(\sim 10-15 \%)$ of WDLPS cells in all primary cultures evaluated $(n=10)$ exhibited a low level of Oil red O positive staining suggesting lipid accumulation; DDLPS cultures $(n=5)$ were negative. Interestingly, a subset of LPS cell strains expressed PPAR $\gamma$ independent of Oil red O positivity or specific histology (WDLPS vs DDLPS; Figure 1d). All WDLPS/DDLPS cell strains examined expressed a variable level CEBP- $\alpha$ per western blot analysis.

\section{A Subset of WDLPS/DDLPS Cells can be Driven to Adipogenic Differentiation}

Next, we evaluated whether WDLPS and/or DDLPS cells retain the capacity to undergo further adipogenic differentiation. Cell strains ( $<10$ passages) were cultured in DM followed by adipocyte growth media as per the recommended human pre-adipocyte differentiation protocol. Similar to the

Table 1 Liposarcoma cell strains

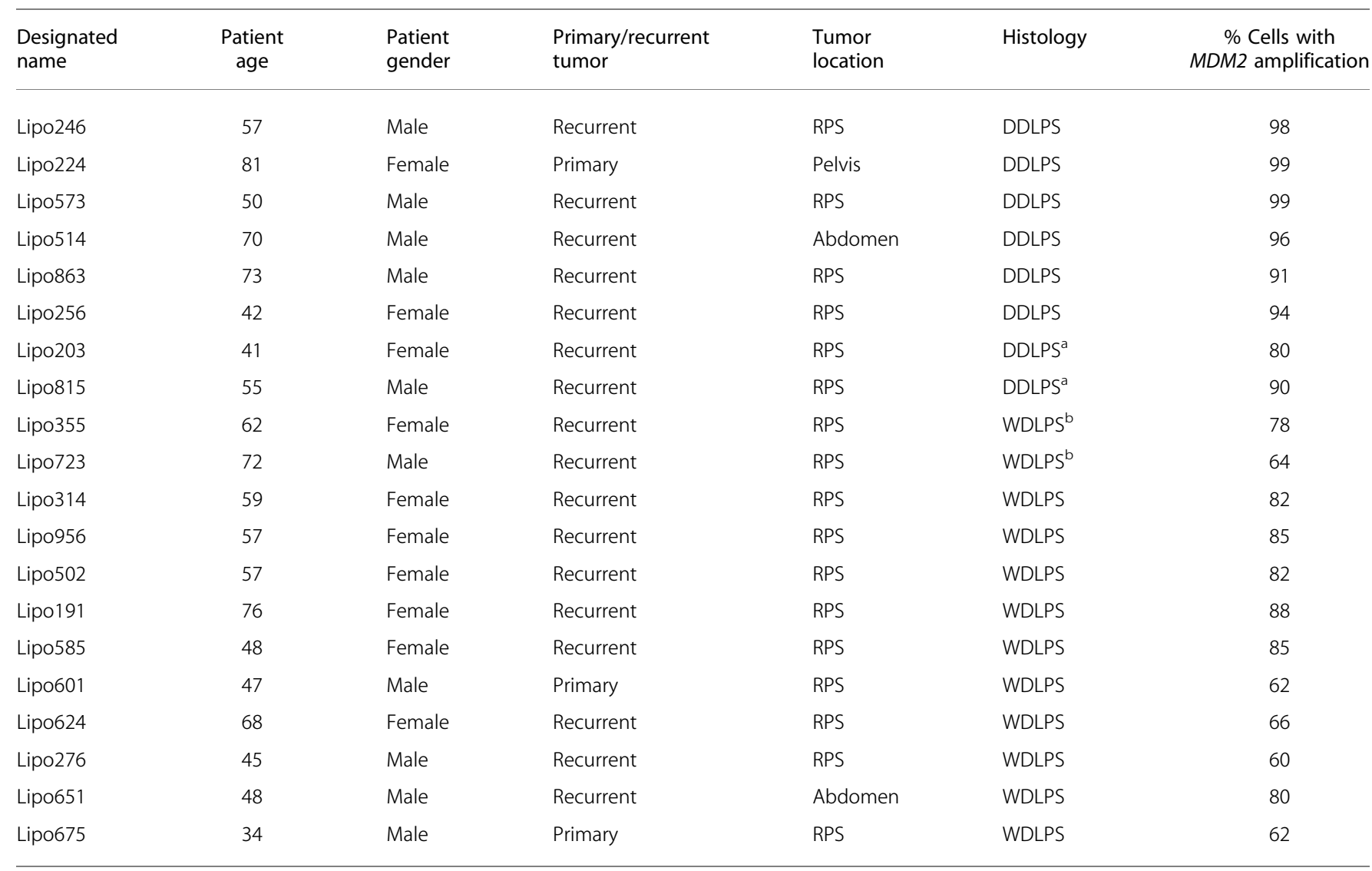

RPS, retroperitoneum.

${ }^{\mathrm{a}}$ Tumor cells isolated from well-differentiated component of a DDLPS.

${ }^{b}$ Tumor cells isolated from a WDLPS tumor from a patient with a history of DDLPS. 
a

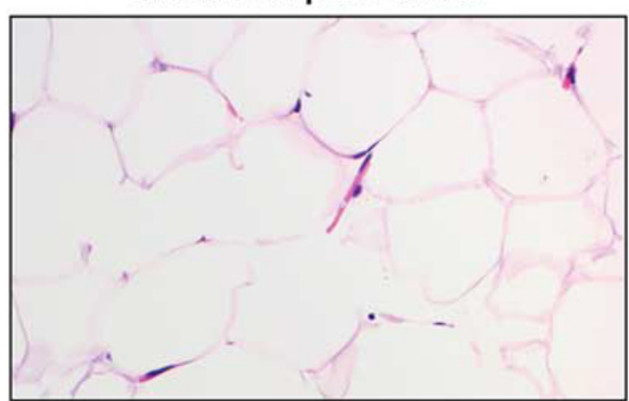

b Pre-adipocytes

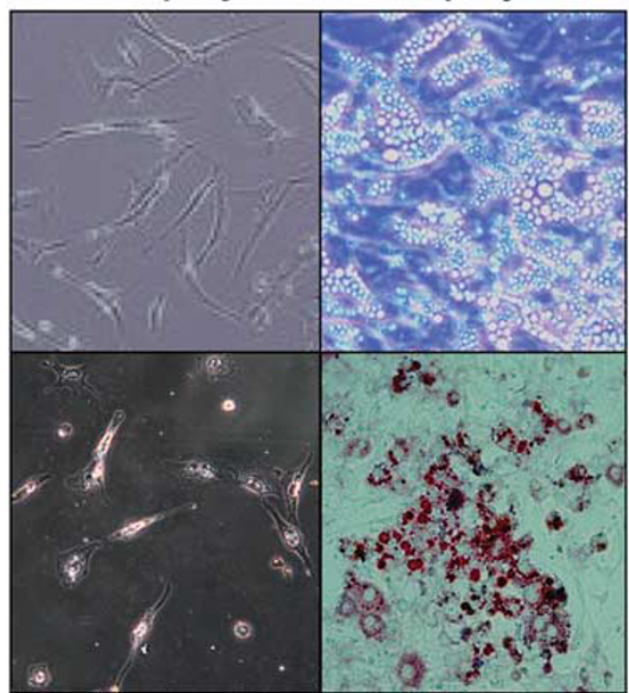

c

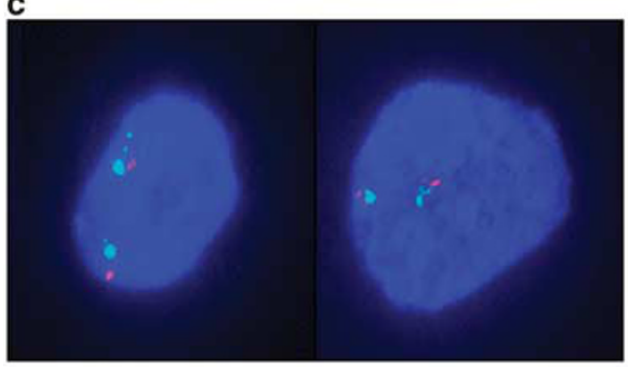

d

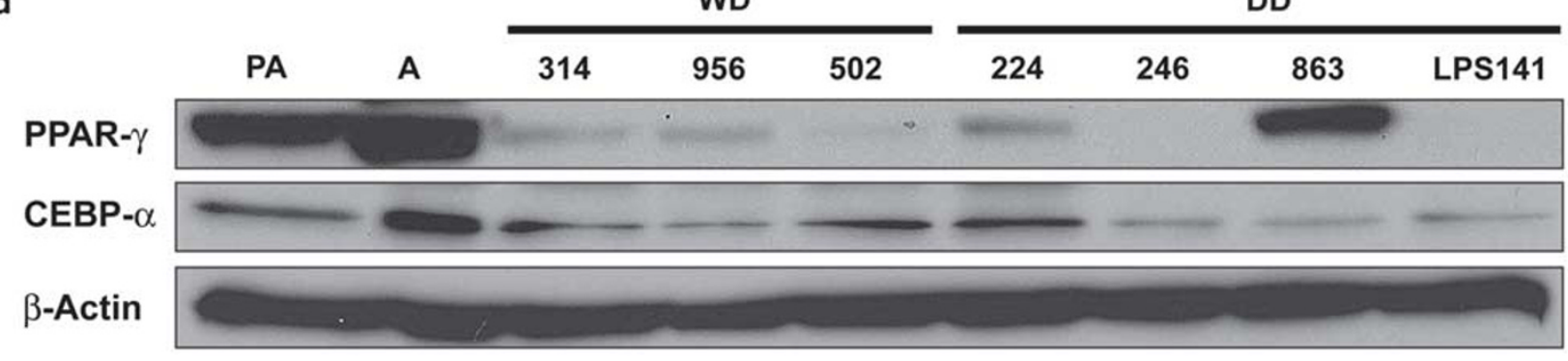

Figure 1 WDLPS and DDLPS cell strains. (a) H+E staining of original tissue/tumor. (b) Morphologic appearance of normal adipogenic-lineage cells and LPS cells and Oil red O staining depicting fat accumulation in normal adipocytes but generally not in WDLPS/DDLPS primary cultured cells (representative cell strains are shown; name depicted in brackets). (c) WDLPS and DDLPS cells (but not pre-adipocytes and adipocytes) exhibit MDM2 amplification (as per FISH analysis). (d) Differentiation of pre-adipocytes (PA) into adipocytes (a) is accompanied by increased PPAR $\gamma$ and CEBP $\alpha$. While lacking lipid accumulation (see b), constitutive, albeit low, expression of PPAR $\gamma$ and CEBP $\alpha$ is observed in a subset of WDLPS/DDLPS cell strains. 
observed effect of DM on normal human pre-adipocytes, three of the five WDLPS primary cultures tested exhibited marked intracellular lipid accumulation accompanied by increased PPAR $\gamma$ and CEBP- $\alpha$ expression (Figures $2 a$ and $b$ ). Similarly, three of four DDLPS cell strains exhibited adipogenic differentiation (Figures 2a and b). All cell strains (either WDLPS or DDLPS) exhibiting the capacity for terminal differentiation expressed a baseline level of PPAR $\gamma$; whereas cells that did not differentiate using DM lacked PPAR $\gamma$ protein expression. Concordantly, the established LPS141 cell line, which lacks PPAR $\gamma$ expression, did not undergo adipogenic differentiation in response to DM.

Interestingly, we observed that all WDLPS cell strains tested exhibit arrested growth after being passaged 12-15 times in culture; till date, we failed to isolate an immortal WDLPS cell line. In contrast, five out of six DDLPS cell strains evaluated demonstrated continuous growth (four of the cell strains have already been growing in culture for over 50 passages, ie, can be designated cell lines); only lipo514 has shown arrested growth after $\sim 20$ passages. Oil red $\mathrm{O}$ staining of WDLPS cultures in late passages demonstrated an increase in fat droplet containing cells and WB demonstrated an increase in expression of adipogenic markers (Figures $2 \mathrm{c}$ and d). These findings possibly suggest that WDLPS undergo terminal adipogenic differentiation in culture. This process was independent of baseline PPAR $\gamma$ expression. MDM2 FISH analysis further demonstrated a statistically significant decrease in the number of MDM2 + cells in latter WDLPS culture passages as compared with early passages (average: $12 \% \pm 3.3$ vs $85 \% \pm 7.1$, respectively, $P<0.05$; Figure 2 c). Micronucleation and nuclear extrusion of amplicons containing MDM2 were observed in these WDLPS cells, leading to decreased number of 12q15 amplicone + cells being present in later culture generations. In contrast, no difference in adipogenic characteristics and percent MDM +-expressing cells was found when DDLPS cultures were evaluated in passage $>35$ (Figures $2 \mathrm{c}$ and $\mathrm{d}$ ).

\section{DDLPS Cells Exhibit Faster Growth, Enhanced Migration and Invasion, and a More Angiogenic Phenotype Compared with WDLPS Cells}

We sought to evaluate the phenotypic characteristics and pro-tumorigenic properties of WDLPS and DDLPS primary cultures. For all experiments, cells in passage $<10$ were used. The WDLPS cell group included cell strains obtained from pure WDLPS samples with no history of DDLPS, the DDLPS group included cell strains obtained from the cellular nonadipogenic component of DDLPS cells. A third group included cell strains obtained from the well-differentiated portions of DDLPS tumors and WDLPS cells obtained from patients with a history of DDLPS; these cells were designated $\mathrm{WD} / \mathrm{DD}$ and were examined separately. For each experiment, a minimum of three different cell strains per group were utilized; results are depicted as an average \pm s.d. DDLPS cells exhibited a significantly shorter doubling time as compared with WDLPS (2 days vs 3.5 days, respectively, $P<0.05$; Figure 3a). Both WDLPS and DDLPS cells demonstrated clonogenic capacity and anchorage-independent growth. However, the average number of colonies formed on plastic and in soft agar was significantly higher for DDLPS cell cultures (WDLPS: $5 \pm 0.9$ and $4 \pm 1 v s$ DDLPS: $33 \pm 5$ and $53 \pm 14$, respectively; $P<0.01$ ). Similarly, DDLPS exhibited significantly enhanced motility and invasion as was seen within $6 \mathrm{~h}$ using modified Boyden chambers $(P=0.006$ and $P=0.0004$, respectively; Figure $3 \mathrm{~b}$ ). In addition, a higher rate of spontaneous apoptosis was identified in WDLPS cells compared with DDLPS cells $(P=0.003$; Figure $3 c)$. Finally, the angiogenic capacity of the tumor cells was evaluated: CM from either WDLPS and DDLPS induced a significantly higher rate of human endothelial cell proliferation compared with regular growth media in vitro $(P=0.63$; Figure $3 \mathrm{~d})$. To also further evaluate potential pro-angiogenic effects in vivo, a gelfoam angiogenesis assay was performed. Gelfoam sponges were incubated in WDLPS-CM and DDLPS-CM (three different cell strains were used for each histology) and implanted subcutaneously into the flanks of SCID mice. An increase in CD31-positive blood vessels was noticed in response to either WDLPS or DDLPS CM; however, a more significant induction was found in response to the latter ( $13 \pm 2.28$ vs $46 \pm 6.57$, respectively, $P<0.01$; Figure $3 \mathrm{~d}$ ). For all parameters evaluated, WD/DD group cells exhibited behavior similar to WDLPS cells (Figure 3). Taken together, our studies demonstrate that DDLPS cells exhibit a more aggressive, pro-tumorigenic phenotype in vitro, recapitulating the clinical scenario.

\section{WDLPS/DDLPS Express an Array of Activated Tyrosine Kinase Receptors}

Next, we asked whether our cell strain model retains the molecular features of WDLPS/DDLPS and as such could be utilized to identify molecular deregulations of potential importance. As an initial confirmation, we evaluated the expression of two markers previously demonstrated to be deregulated in WDLPS/DDLPS: CDK4 and JUN. As shown in Figure 4a, CDK4 was found to be markedly overexpressed in LPS cells compared with adipocytes; as anticipated, no significant difference in expression level was found between WDLPS and DDLPS cells. Similarly, JUN overexpression was also identified in LPS cells. Furthermore, higher JUN and phospho-JUN expression levels were found in DDLPS cells compared with WDLPS cells $(P=0.03)$. With the recent emergence of TKRs as targets that are highly susceptible to molecular-based therapies, we next sought to evaluate such receptors in LPS cells (Figure 4b). Eight receptors for which small molecule inhibitors are currently available were evaluated: EGFR expression was identified in all LPS cells but also in adipocytes. MET, AXL, and IGFR were found to be overexpressed in LPS compared with both adipocytes and pre-adipocytes. All LPS cells were found to express PDGFRs, but pre-adipocytes exhibited a higher level of expression. All 


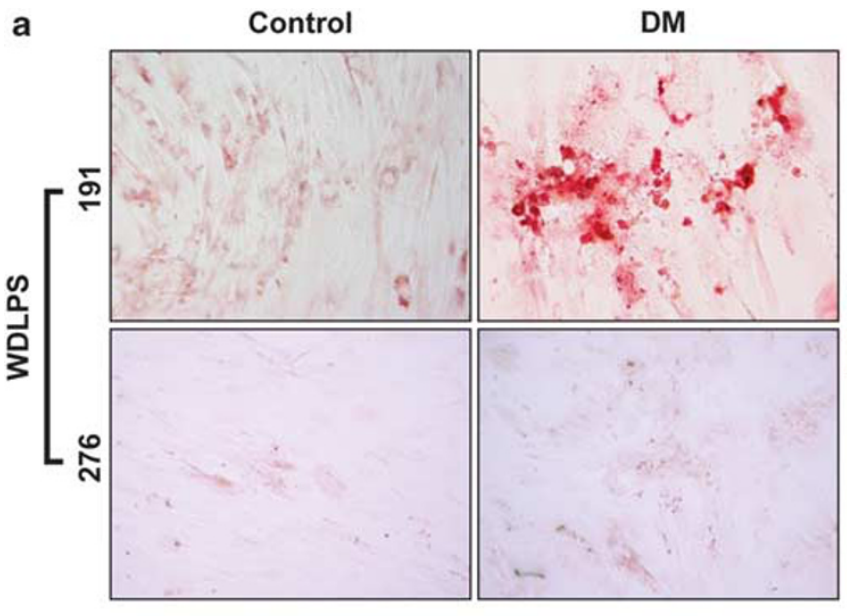

b

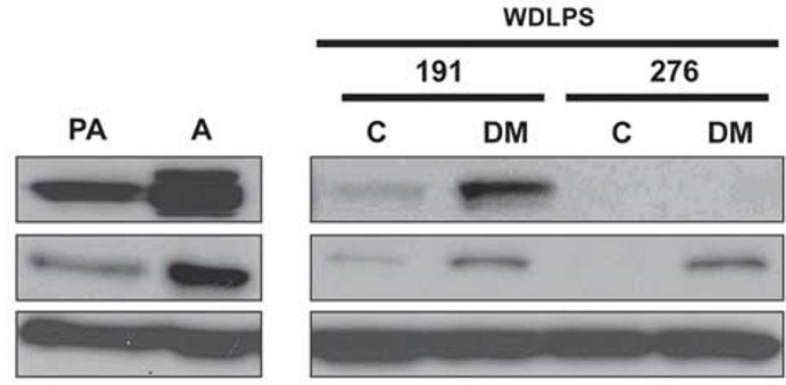

C

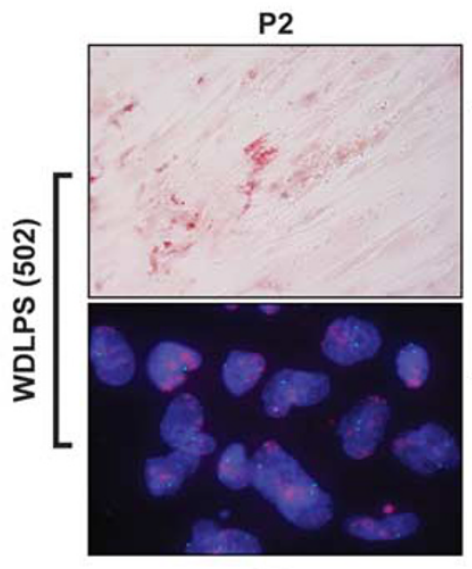

P2

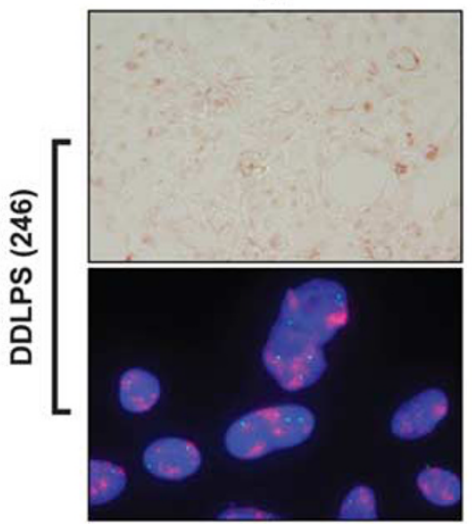

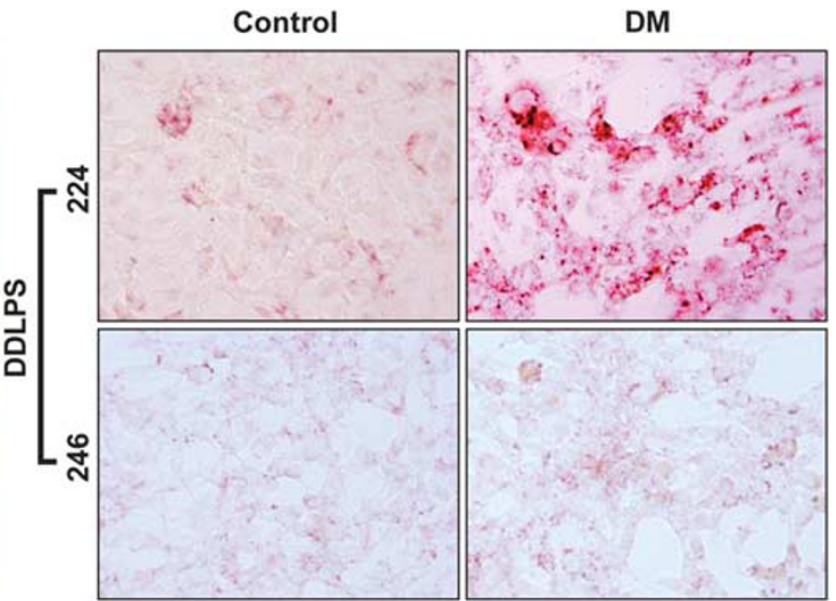
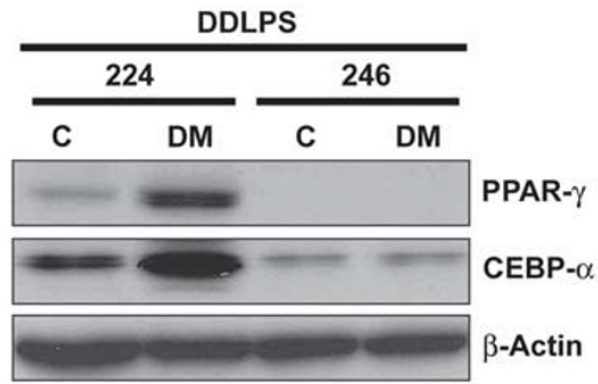

d

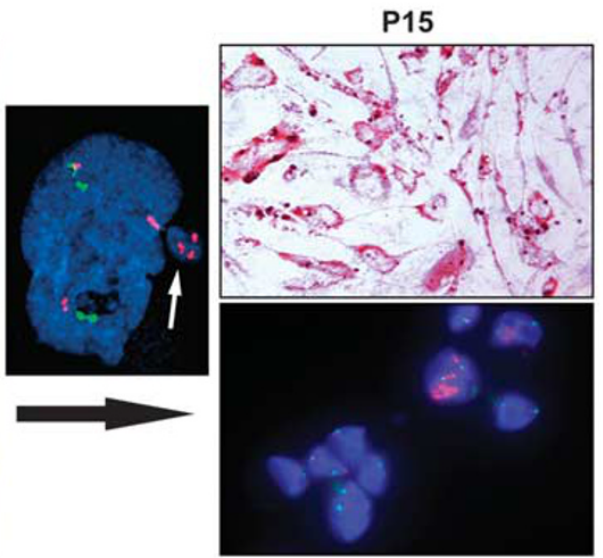

P35

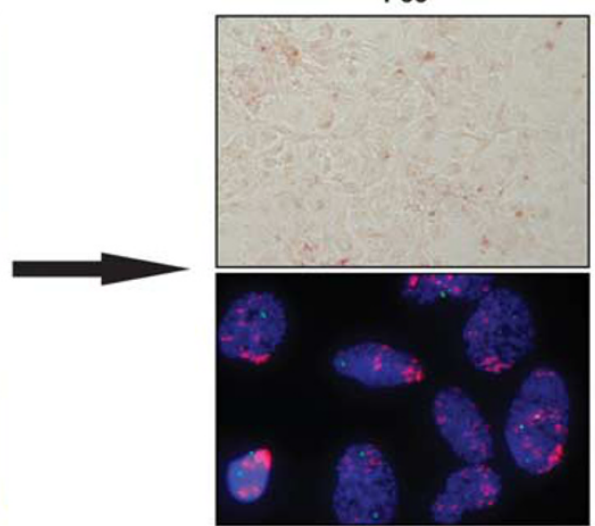

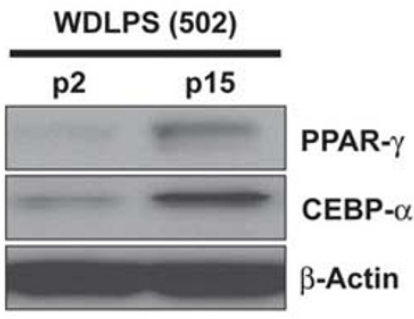

$\frac{\text { DDLPS (246) }}{\text { p2 }}$

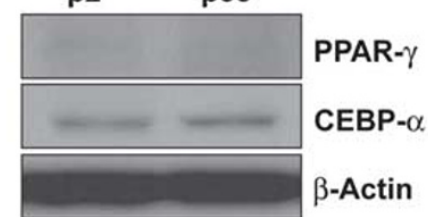


evaluated cells (including normal cells) were negative for HER2 and KIT (data not shown).

\section{DDLPS Cells Exhibit Reproducible Growth in SCID Mice}

Finally, we sought to evaluate the growth patterns of WDLPS/DDLPS in vivo with the goal of confirming the tumorigenic differences identified in vitro seeking also to establish reproducible mouse models that will be essential for future LPS studies. Cell strains (WDLPS: Lipo314, Lipo956, Lipo601, Lipo675, WD/DD: Lipo203, and DDLPS: Lipo246, Lipo224, Lipo863; $2 \times 10^{6}$ cells/mouse) at culture passage 2-6 were injected subcutaneously and/or intraperitoneally into hairless SCID mice that were then followed for up to 8 months. None of the WDLPS as well as the WD/DD cell lines tested developed tumors. In contrast, all three DDLPS cell strains resulted in tumor development with varying tumor take rates, latency periods, and growth rates. The most pronounced growth was found for the Lipo246 cell, exhibiting a tumor take of $90 \%$, a latency period of 10-14 days, and growth to $\sim 1.5 \mathrm{~cm}$ tumor by $8 \pm 2$ weeks (Figure $5 \mathrm{a}$ ). A tumor take of $40-60 \%$ was found for the other two cell strains tested, with latency periods ranging between 4 and 6 months and a tumor growth to $1.5 \mathrm{~cm}$ noticed by $10-12$ months after initial injection. $\mathrm{H}+\mathrm{E}$ staining of xenograft tissue samples demonstrated a histological appearance resembling the original tumor, and MDM2 FISH demonstrated MDM2 amplification in vivo (Figure 5a). Tumor cell proliferation and angiogenesis were demonstrated via Ki-67 and CD31 immunostaining, respectively (Figure 5b). TKR expression in vivo correlated with the expression noted in culture. Furthermore, in all cases, fresh tumor tissue was processed, and recycled tumor cells were isolated and confirmed to be $M D M 2+$. In addition, Lipo246 at culture passage $>40$ (ie cell line) has been evaluated, demonstrating a highly reproducible and fast growth rate as per above, suggesting that this cell line can be utilized for therapeutic experiments. Taken together, these data confirm that DDLPS exhibits a more pronounced tumorigenic phenotype and that our newly developed cell lines are novel bioresources that can be used for anti-LPS drug testing in vivo.

\section{DISCUSSION}

Current lack of relevant human LPS cell lines and animal models limits our capacity to translate clinical and tissuebased LPS-related observations into comprehensive molecular and mechanistic insights and, most importantly, to identify and test novel therapeutic strategies specifically targeting LPS. As a consequence, inclusion of LPS patients in clinical trials usually relies almost exclusively on subjective tissue-based observations, and does not utilize extensive preclinical (molecular derived) evaluation criteria as is now increasingly common in other type of cancers. In our era of evidence-based medicine, the availability of molecularly informative, clinically relevant cancer models is crucial. Towards that end, we have shown that isolated human WDLPS/DDLPS cell strains/lines and xenograft animal models recapitulate clinical LPS behavior and retain the molecular deregulations of their tumor of origin. DDLPS cells exhibit a significantly more tumorigenic and aggressive phenotype. Consequently, the cellular and xenograft models described here can serve as particularly incisive tools for the investigation of liposarcomagenesis, dedifferentiation, and tumor progression.

Several published studies have shown that the WD component of DDLPS is molecularly similar to the DD fraction of the tumor and can possibly be distinguished from pure WDLPS via aCGH and gene expression profiling. ${ }^{23-25}$ In our study, the WD cell strains isolated from DDLPS (ie WD/DD cells) were found to functionally behave like the pure WDLPS cell strains. The small number of samples in the WD/DD group precludes making affirmative conclusions, and a larger cohort of cell strains is needed to validate this initial insight. Additional studies are needed to extensively dissect the genetic and epigenetic molecular deregulations governing each of the three LPS cell strain subsets and would hopefully be able to resolve the possible genotypic/molecular vs pheonotypic discrepancy highlighted above.

Although the exact WDLPS/DDLPS cell of origin has not yet been defined, pathology-based studies strongly suggest an adipogenic-lineage origin. Furthermore, several lines of circumstantial evidence suggest that DDLPS may represent a progression of WDLPS. ${ }^{26,27}$ It has recently been proposed that an initial genetic change, ie, amplification of chromosome 12q12-15 occurs within a cell in the adipogenic lineage, resulting in differentiation stage arrest that morphologically appears as WDLPS. ${ }^{17,25,28}$ Upon further accumulation of genetic changes, the differentiation potential of the same cells that gave rise to the WDLPS is further significantly impaired, giving rise to what is termed a "dedifferentiated" tumor, DDLPS. As shown in our study, and in support of previously published data, ${ }^{22,29}$ all WDLPS and DDLPS primary cell cultures contain the MDM2 amplicon. However, the forces driving dedifferentiation are still unknown and might be

Figure 2 WDLPS and DDLPS cells retain, at least in part, their capacity for adipogenic differentiation. (a) A subset of WDLPS and DDLPS cells cultured in differentiation media (for 3 days) followed by adipocyte nutrition media (for 12 days) demonstrate increase in fat lipids, whereas other WDLPS/DDLPS cells do not, representative cell strains are shown. (b) Similarly, an increase in adipogenic markers is observed (WB) in cells exhibiting adipogenic differentiation. Interestingly, all cells capable of differentiation after culture in differentiation media expressed a basal level of PPAR $\gamma$, representative cell strains are shown. (c) WDLPS cells exhibit arrested growth in culture after $>12$ passages that is accompanied by lipid accumulation, whereas DDLPS cells demonstrate continuous growth and no lipid accumulation. Interestingly, exclusion of micronuclei containing amplified regions and a gradual decrease in the number of MDM2 + cells is observed in WDLPS primary cultures (representative cell strains are shown; name depicted in brackets). (d) Growth-arrested WDLPS cells exhibit an increase in PPAR $\gamma$ and CEBP $\alpha$ expression (representative cell strains are shown; name depicted in brackets). 
further unraveled using the model described above. Interestingly, our data suggest that at least of subset of WDLPS and DDLPS cells retain the capacity for terminal adipogenic differentiation. In WDLPS cells, this process occurs spontaneously for all cells tested when grown in culture or in response to DM in cells that express baseline levels of PPAR $\gamma$.

a
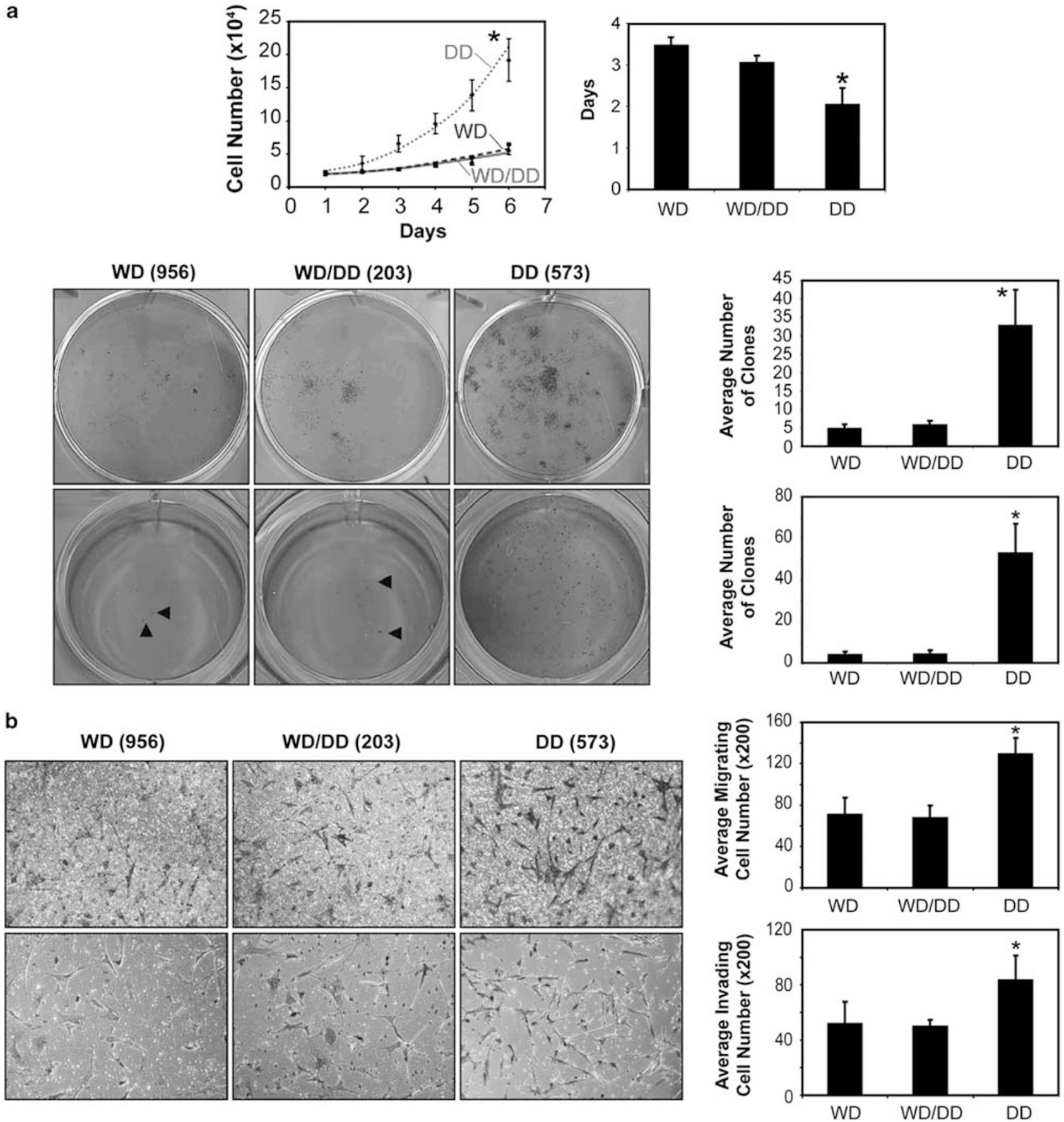

Figure 3 DDLPS cells exhibit a more aggressive phenotype as compared with WDLPS cells. (a) DDLPS cells (DD) exhibit a statistically significant enhanced growth (upper left panel), a shorter doubling time (upper right panel), increased clonogenicity (middle panel), and anchorage-independent growth (lower panel) as compared with WDLPS cells (WD) and WDLPS cells from DDLPS patients (WD/DD; $\left.{ }^{\star} P<0.05\right)$. (b) DD cells exhibit statistically significantly enhanced migration (upper panels) and invasion (lower panels) as compared with WD and WD/DD cells $\left({ }^{*} P<0.05\right)$. (c) DD cells exhibit a statistically significantly decreased level of spontaneous apoptosis compared with WD and WD/DD cells ( ${ }^{*} P<0.05$ ). (d) HDMVC and HUVECs grown in conditioned media (CM) obtained from WD, WD/DD, and DD exhibit enhanced proliferation compared with cells grown in control serum-free media (upper panel; ${ }^{*} P<0.05$ ). In vivo gelfoam assay demonstrated increase in blood vessel formation in response to WD, WD/DD, and DD CM, which was most pronounced in response to the latter $\left({ }^{*} P<0.05\right)$ compared with gelfoam suspended in control serum-free media (red $\left.=C D 31\right)$ (representative cell strains are shown in all panels; name depicted in parentheses). 
c

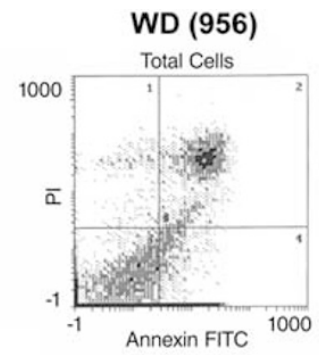

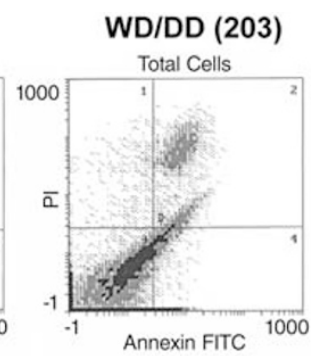
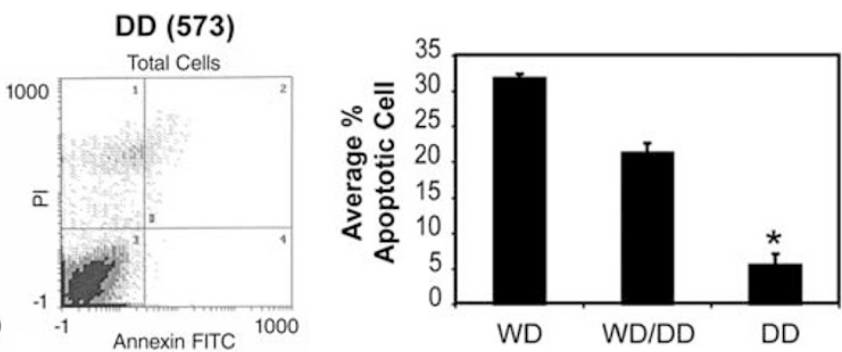

d
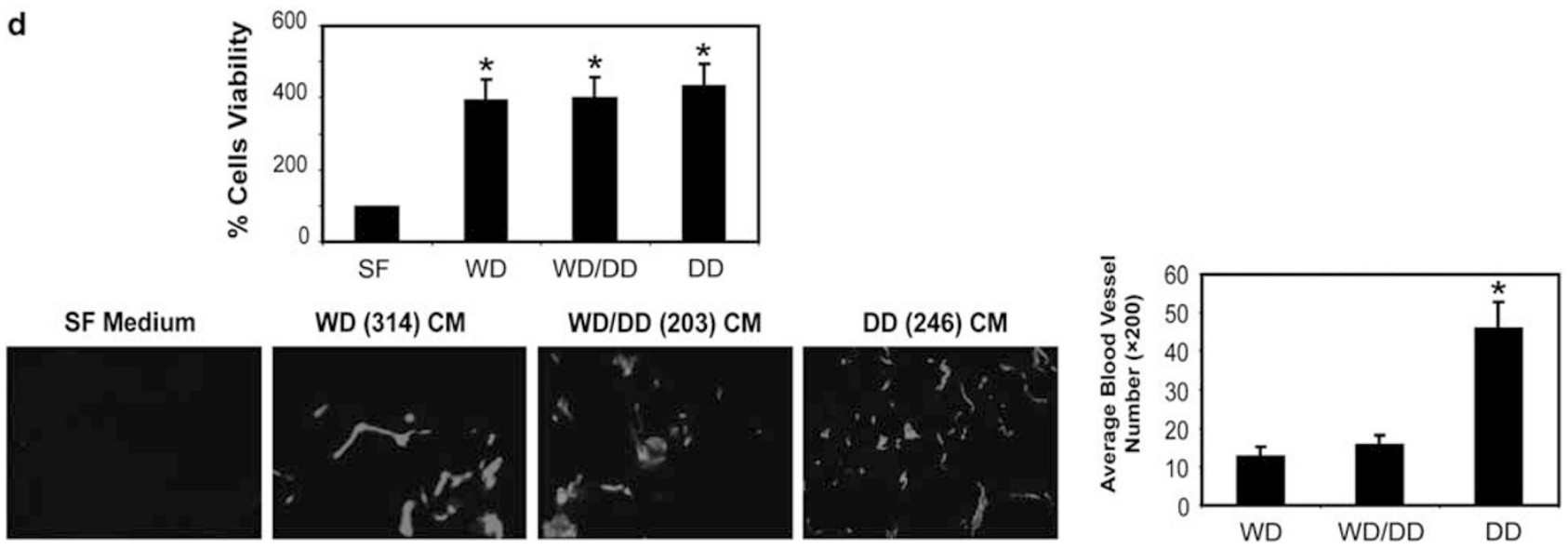

Figure 3 Continued.

a
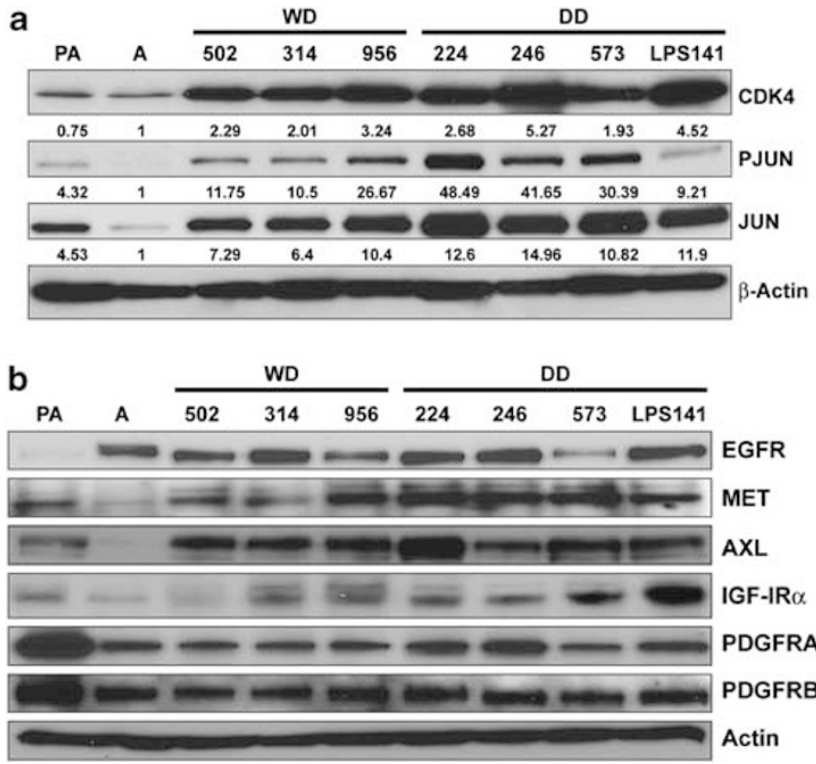

Figure 4 Molecular deregulations are maintained in WDLPS/DDLPS cell strains. (a) CDK4 is markedly overexpressed in LPS cells compared with adipocytes. Higher JUN and phospho-JUN expression levels are found in DDLPS cells compared with WDLPS cells $(P=0.03$; relative protein expression levels were determined via densitometry and are depicted below each WB). (b) WB analyses depicting the expression of a panel of TKRs in pre-adipocytes (PA), adipocytes (A), WDLPS cells (WD), and DDLPS cells (DD).
Intriguingly, spontaneous terminal adipogenic differentiation was accompanied by the extrusion of micronuclei containing the MDM2 amplicon. This finding is in accordance with recently published data, suggesting that selective elimination of amplified sequences correlates with spontaneous adipocytic differentiation in liposarcoma. ${ }^{30}$ The mechanisms of this process, and whether this is a cell culture-based observation or an actual phenomenon occurring in vivo and potentially contributing to the predominant adipogenic content observed in human WDLPS tumors is uncertain and should be further investigated. In contrast, DDLPS cells sustain immortal growth in culture and do not spontaneously differentiate. However, when grown in adipogenic $\mathrm{DM}$, a varying degree of fat accumulation is observed in cells expressing PPAR $\gamma$. This differential effect can possibly be explained by the presence of a thiazolidinedione class PPAR $\gamma$ ligand (ciglitazone) in differentiation medium, consequently affecting only PPAR $\gamma$-expressing cells. This finding is of potential major clinical implication, suggesting that LPS can be driven to a more differentiated state. Along the same line, previous studies have suggested that strategies activating PPAR $\gamma$ might induce re-differentiation in LPS. ${ }^{31}$ However, the study of troglitazone, a PPAR $\gamma$ agonist, in a phase II clinical trial for LPS patients failed to achieve any objective clinical responses. ${ }^{32}$ This possibly indicates that blockade of this single pathway is insufficient to induce significant effect 
a
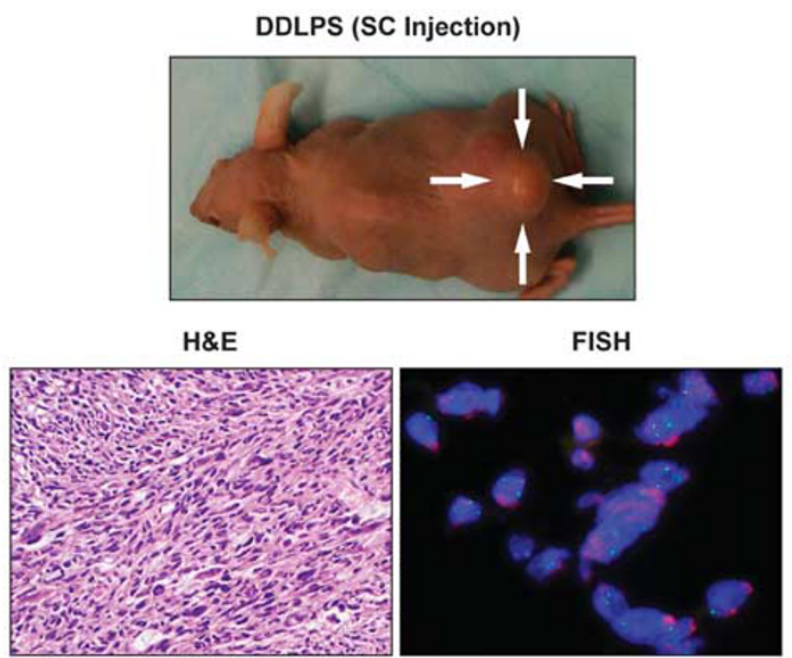

DDLPS (IP Injection)

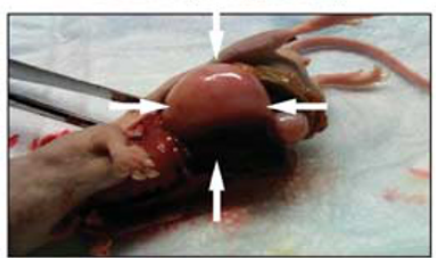

$H \& E$

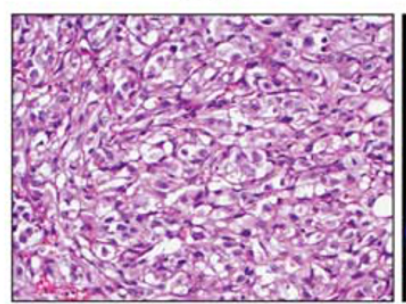

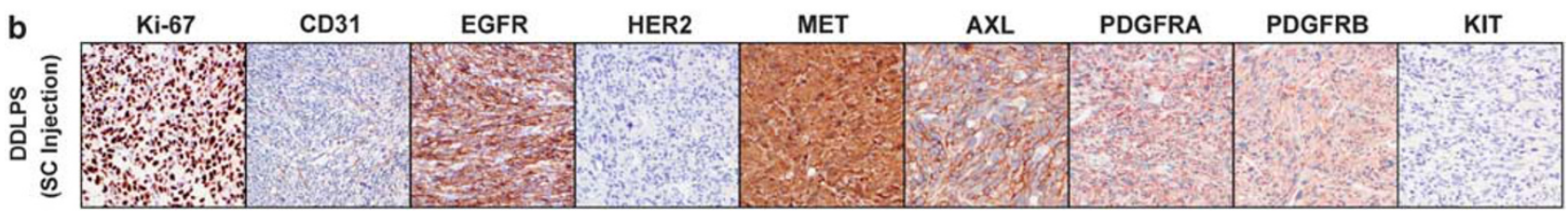

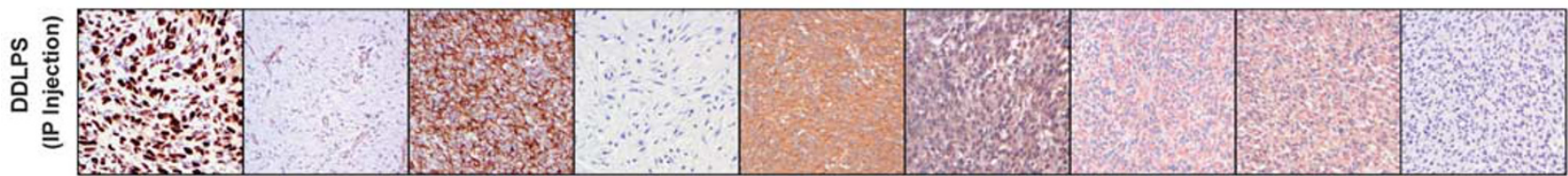

Figure 5 A human xenograft DDLPS mouse model. (a) DDLPS cells ( $\left.2 \times 10^{6} / \mathrm{mouse}\right)$ reproducibly grow in hairless SCID mice after subcutaneous (SC; left panel, LPS246 xenograft is shown as an example) or intraperitoneal (IP; right panel, Lipo224 xenograft is shown as an example) injection. $H+E$ staining demonstrating high-grade DDLPS and MDM2 + in tumor xenografts. (b) IHC analysis depicting enhanced proliferation (Ki-67) and angiogenesis (CD31) in DDLPS (lipo246-upper panel, lipo224-lower panel). Concordant with in vitro findings (see Figure 4b above), DDLPS express high levels of EGFR, MET, AXL, and PDGFRs. No HER2 and KIT expression could be identified.

in vivo; studies using our described model will enable the identification of additional differentiation-relevant targets.

Several potential therapeutic targets for the treatment of WDLPS and/or DDLPS have recently been proposed including MDM2, CDK4, and JUN; $;^{16,27-35}$ the deregulation of these targets was confirmed in our cellular model. Nutlin-3A, an MDM2 inhibitor, has recently been shown to have antiLPS effects in vitro and is currently being tested in human clinical trials. ${ }^{16,18}$ Amplification of CDK4 has been demonstrated in $\sim 90 \%$ of WDLPS/DDLPS; ${ }^{34}$ several CDK4-specific inhibitors have recently been developed and are currently tested in various human malignancies. ${ }^{36} \mathrm{JUN}$ amplification and overexpression has been suggested as a mechanism of WDLPS progression to DDLPS. ${ }^{19,36}$ Our study further validated this observation, demonstrating a significantly higher JUN and phosphor-JUN in DDLPS cells as compared with WDLPS cells and normal adipocytes. Inhibitors of the JUN pathway are currently under development ${ }^{37}$ and should be further tested for their efficacy in DDLPS. In recent years, an important role for TKRs has emerged as novel candidates easily amenable to therapeutic targeting; HER-2 in breast cancer and KIT in gastrointestinal tumors are two clinically relevant examples. Here, we found that WDLPS and DDLPS cells overexpress several TKRs, including EGFR, MET, AXL, and IGFR, all of which are targets of currently available small molecule inhibitors. However, these TKRs have yet to be tested in the context of LPS. Regarding EGFR, we identified its expression in both adipocytes and LPS cells. Interestingly, recent studies have identified that EGF-induced activation of the EGFR can promote adipogenesis cells when administered in low concentrations $(<1 \mathrm{nM}) ;^{38}$ however, at higher doses, EGF inhibits this differentiation. ${ }^{39}$ Further studies to evaluate this intriguing phenomenon in the context of LPS are currently ongoing. Hopefully, the availability of new models such as that described in this report will provide a heretofore critically lacking investigative platform upon which to examine LPS molecular regulatory machinery, thereby setting the stage for preclinical testing of the above-mentioned inhibitors alone and in novel therapeutic combinations as anti-LPS strategies.

In summary, LPS-related bioresources developed here can be utilized for the comprehensive investigation of WDLPS/ 
DDLPS. Further studies of initial molecular insights described are currently ongoing and will hopefully result in the development of new therapeutic strategies for the clinical management of patients harboring these poor prognosis malignancies.

\section{ACKNOWLEDGEMENTS}

We would like to thank Dr Jonathan Fletcher (Brigham and Women's, Boston, MA, USA) for providing us the LPS141 cell line. We appreciate the expert assistance provided by Mr Paul Cuevas in the preparation and submission of this manuscript, and Ms Kim Vu is thanked for her aid in figure preparation. We highly appreciate the philanthropic support of the Lobo, Margolis, and Jackson families. This manuscript was supported in part by an $\mathrm{NIH} / \mathrm{NCl}$ RO1CA138345 grant (to DL) and a Liddy Shriver seed grant (to $\mathrm{DL}$ ). The MD Anderson Cancer Center cell line characterization Core Facility is supported by an NCl Cancer Center Support Grant (CA\#16672).

\section{DISCLOSURE/CONFLICT OF INTEREST}

The authors declare no conflict of interest.

1. Dei Tos AP, Pedeutour F. Atypical lipomatous tumor/well differentiated liposarcoma and dedifferentiated liposarcoma. In: Fletcher CDM Unni KK, Mertens F (eds) Pathology and Genetics of Tumours of Soft Tissue and Bone. IARC: Lyon, 2002, p 35-39.

2. Lahat G, Anaya DA, Wang X, et al. Resectable well-differentiated versus dedifferentiated liposarcomas: two different diseases possibly requiring different treatment approaches. Ann Surg Oncol 2008; 15:1585-1593.

3. Evans HL. Liposarcoma: a study of 55 cases with a reassessment of its classification. Am J Surg Pathol 1979;3:507-523.

4. Evans HL. Atypical lipomatous tumor, its variants, and its combined forms: a study of 61 cases, with a minimum follow-up of 10 years. Am J Surg Pathol 2007;31:1-14.

5. Weiss SW, Goldblum JR. Liposarcoma. In: Weiss SW, Goldblum JR (eds) Enzinger and Weiss's Soft Tissue Tumors. Mosby: St Louis, 2008, p 477-516.

6. Henricks WH, Chu YC, Goldblum JR, et al. Dedifferentiated liposarcoma. A clinicopathologic analysis of 155 cases with a proposal for an expanded definition of dedifferentiation. Am J Surg Pathol 1997; 21:271-281.

7. McCormick D, Mentzel T, Beham A, et al. Dedifferentiated liposarcoma. Clinicopathologic analysis of 32 cases suggesting a better prognostic subgroup among pleomorphic sarcomas. Am J Surg Pathol 1994; 18:1213-1223.

8. Kimberly MD, Cristina RA, Samuel S. Diagnosis and management of lipomatous tumors. J Surg Oncol 2008;97:298-313.

9. Takahira $T$, Oda $Y$, Tamiya $S$, et al. Alterations of the RB1 gene in dedifferentiated liposarcoma. Mod Pathol 2005;18:1461-1470.

10. Micci F, Bjerkehagen B, Heim S. Pairwise comparison of genomic imbalances between primary and recurrent well differentiated liposarcomas. Cancer Genet Cytogenet 2007;178:163-167.

11. Pedeutour F, Forus A, Coindre JM, et al. Structure of the supernumerary ring and giant rod chromosomes in adipose tissue tumors. Genes Chromosomes Cancer 1999;24:30-41.

12. Italiano A, Blanchini L, Keslair F, et al. HMGA2 is the partner of MDM2 in well-differentiated and dedifferentiated liposarcomas whereas CDK4 belongs to a distinctive inconsistent amplicon. Int J Cancer 2008; 122:2233-2241.

13. Momand J, Wu HH, Dasgupta G. MDM2-master regulator of the $\mathrm{p} 53$ tumor suppressor protein. Gene 2000;242:15-29.

14. Ortega S, Malumbres M, Barbacid M. Cyclin D-dependent kinases, INK4 inhibitors and cancer. Biochim Biophys Acta 2002;1602:73-87.

15. Fritz B, Schubert F, Wrobel G, et al. Microarray-based copy number and expression profiling in dedifferentiated and pleomorphic liposarcoma. Cancer Res 2002;62:2993-2998.

16. Singer S, Socci ND, Ambrosini G, et al. Gene expression profiling of liposarcoma identifies distinct biological types/subtypes and potential therapeutic targets in well-differentiated and dedifferentiated liposarcoma. Cancer Res 2007;67:6626-6636.
17. Matushansky I, Hernando E, Socci ND, et al. A developmental model of sarcomagenesis defines a differentiation-based classification for liposarcomas. Am J Path 2008;17:1069-1080.

18. Debrock G, Vanhentenrijk V, Sciot $R$, et al. A phase II trial with rosiglitazone in liposarcoma patients. Br J Cancer 2003;89:1409-1412.

19. Snyder EL, Sandstrom DJ, Law K, et al. c-Jun amplification and overexpression are oncogenic in liposarcoma but not always sufficient to inhibit the adipocytic differentiation programme. J Pathol 2009;218:292-300.

20. Ramírez-Zacarías JL, Castro-Muñozledo F, Kuri-Harcuch W. Quantitation of adipose conversion and triglycerides by staining intracytoplasmic lipids with Oil red O. Histochemistry 1992;97:493-497.

21. Lahat $\mathrm{G}$, Zhu QS, Huang $\mathrm{KL}$, et al. Vimentin is a novel anti-cancer therapeutic target; insights from in vitro and in vivo mice xenograft studies. PLoS One 2010;5:e10105.

22. Weaver J, Downs-Kelly E, Goldblum JR, et al. Fluorescence in situ hybridization for MDM2 gene amplification as a diagnostic tool in lipomatous neoplasms. Mod Pathol 2008;21:943-949.

23. Chibon F, Mariani O, Derré J, et al. A subgroup of malignant fibrous histiocytomas is associated with genetic changes similar to those of well-differentiated liposarcomas. Cancer Gen Cytogenet 2002;139: 24-29.

24. Shimoji $\mathrm{T}$, Kanda $\mathrm{H}$, Kitagawa $\mathrm{T}$, et al. Clinico-molecular study of dedifferentiation in well-differentiated liposarcoma. Biochem Biophys Res Commun 2004;314:1133-1140.

25. Horvai $A E$, DeVries $S$, Roy $R$, et al. Similarity in genetic alterations between paired well-differentiated and dedifferentiated components of dedifferentiated liposarcoma. Mod Pathol 2009;22:1477-1488.

26. Mai HE, Aisner S, Benevenia J, et al. Epigenetic alteration of p16INK4a gene in dedifferentiation of liposarcoma. Pathol Res Pract 2009;205: 386-394.

27. Coindre JM, Pédeutour F, Aurias A. Well-differentiated and dedifferentiated liposarcomas. Virchows Arch 2010;456:167-179.

28. Trahan S, Erickson-Johnson MR, Rodriguez $F$, et al. Formation of the 12q14-q15 amplicon precedes the development of a welldifferentiated liposarcoma arising from a nonchondroid pulmonary hamartoma. Am J Surg Pathol 2006;30:1326-1329.

29. Aleixo PB, Hartmann AA, Menezes IC, et al. Can MDM2 and CDK4 make the diagnosis of well differentiated/dedifferentiated liposarcoma? An immunohistochemical study on 129 soft tissue tumours. J Clin Pathol 2009;62:1127-1135.

30. Hélias-Rodzewicz Z, Pédeutour F, Coindre JM, et al. Selective elimination of amplified CDK4 sequences correlates with spontaneous adipocytic differentiation in liposarcoma. Genes Chromosomes Cancer 2009;48:943-952.

31. Tontonoz $\mathrm{P}$, Singer $\mathrm{S}$, Forman $\mathrm{BM}$, et al. Terminal differentiation of human liposarcoma cells induced by ligands for peroxisome proliferator-activated receptor gamma and the retinoid $\mathrm{X}$ receptor. Proc Natl Acad Sci 1997;94:237-241.

32. Müller $C R$, Paulsen EB, Noordhuis $P$, et al. Potential for treatment of liposarcomas with the MDM2 antagonist Nutlin-3A. Int J Cancer 2007;121:199-205.

33. Pires de Camargo V, van de Rijn M, Maestro R, et al. Other targetable sarcomas. Semin Oncol 2009;36:358-371.

34. Italiano A, Bianchini L, Gjernes E, et al. Clinical and biological significance of CDK4 amplification in well-differentiated and dedifferentiated liposarcomas. Clin Cancer Res 2009;15:5696-5703.

35. Mariani $\mathrm{O}$, Brennetot $\mathrm{C}$, Coindre JM, et al. JUN oncogene amplification and overexpression block adipocytic differentiation in highly aggressive sarcomas. Cancer Cell 2007;11:361-374.

36. Michaud K, Solomon DA, Oermann E, et al. Pharmacologic inhibition of cyclin-dependent kinases 4 and 6 arrests the growth of glioblastoma multiforme intracranial xenografts. Cancer Res 2010;70:3228-3238.

37. Shrotriya S, Kundu JK, Na HK, et al. Diallyl trisulfide inhibits phorbol ester-induced tumor promotion, activation of AP-1, and expression of COX-2 in mouse skin by blocking JNK and Akt signaling. Cancer Res 2010;70:1932-1940.

38. Harrington $\mathrm{M}$, Pond-Tor S, Boney $\mathrm{CM}$. Role of epidermal growth factor and ErbB2 receptors in 3T3-L1 adipogenesis. Obesity 2007; 15:563-571.

39. Lee JS, Suh JM, Park HG, et al. Heparin-binding epidermal growth factorlike growth factor inhibits adipocyte differentiation at commitment and early induction stages. Differentiation 2008;76:478-487. 